\section{GENE TRANSFER AS A NEW MODE OF VACCINATION: IMPLICATIONS FOR HCV}

Ulmer JB, Donnelly JJ, Parker SE, Rhodes GH, Felgner PL, Dwarki VJ, Gromkowski SH, Deck RR, DeWitt CM, Friedman A, Hawe LA, Leander KR, Martinez D, Perry HC, Shiver JW, Montgomery DL, Liu $M A$. Heterologous protection against influenza by injection of DNA encoding viral protein. Science 1993;259: 1745-1749.

\section{ABSTRACT}

Cytotoxic T lymphocytes (CTLs) specific for conserved viral antigens can respond to different strains of virus, in contrast to antibodies, which are generally strain-specific. The generation of such CTLs in vivo usually requires endogenous expression of the antigen, as occurs in the case of virus infection. To generate a viral antigen for presentation to the immune system without the limitations of direct peptide delivery or viral vectors, plasmid DNA encoding influenza A nucleoprotein was injected into the quadriceps of BALB/c mice. This resulted in the generation of nucleoproteinspecific CTLs and protection from a subsequent challenge with a heterologous strain of influenza $A$ virus, as measured by decreased viral lung titers, inhibition of mass loss, and increased survival.

\section{COMMENTS}

This work presents two novel concepts. The first is the use of naked plasmid DNA in gene therapy rather than DNA packaged into some more sophisticated complex such as a virus or lipid. The second is an effective vaccine that activates cell-mediated immunity rather than humoral immunity.

The goal in gene-transfer research has been to get the gene you would like into a sufficient number of cells and have the desired protein expressed in sufficient quantity to have a desired therapeutic benefit. Strategies for this have included packaging the gene in retroviral vectors, in adenoviral vectors and in cationically charged lipids (liposomes) called Lipofectin (1). Another level of complexity is encountered in targeting the vector to the desired tissue. Vectors can be given directly to the animal tissues intravenously; intraarterially; as an aerosol; or through potential receptor ligands such as lysine-bound asialoglycosides, which are taken up by the liver by way of the asialoglycoprotein receptor. Alternatively, target cells (e.g., lymphocytes, hepatocytes, tumor cells) can be removed, transfected with the vector ex vivo and then returned to the animal. But Jon Wolff at the

Hepatology $1993 ; 18 ; 696-702$

$31 / 8 / 49178$
University of Wisconsin and investigators from Vical, Inc. (San Diego, CA), happened upon a much more direct mode of gene transfer (2). These investigators found that naked DNA in the form of a plasmid expression vector could be simply injected into animal muscle and the DNA would be taken up by the cells and expressed. They discovered this quite by accident when Wolff et al. used plasmid DNA with a reporter gene as a negative control in their gene-transfer experiments (3). Much to their surprise, they found that muscles injected with the negative control not only took up the DNA but also expressed high levels of the reporter protein. The next step was to devise some model in which this technology could be used to not express just a reporter gene but some gene that would potentially be of benefit to the animal and then actually demonstrate that benefit. Ulmer and colleagues accomplished this by using an influenza gene to induce immunity and protect recipient mice from a lethal dose of influenza A.

To understand why gene-transfer technology was essential in the study under comment to induce immunity against influenza, a word on antigen processing is in order. Antigen processing and, therefore, the immune response to vaccines are generally divided into two systems, although considerable crossover exists (4). The first is the class II major histocompatibility complex (MHC) system or the exogenous pathway. Here antigens that invade the body are taken up by phagocytosis or endocytosis into cells that express MHC class II molecules, such as macrophages or neutrophils. The antigen is processed in the endosome, where it binds the MHC molecule. MHC molecules are similar to antibodies in that they recognize distinct epitopes, but whereas antibody recognition is dependent in part on the secondary and tertiary structure of the antigen, MHC molecules recognize processed antigens that are cleaved by proteosomes into oligopeptides of 8 to 12 amino acids. This complex is then transported to the cell surface, where it binds MHC class II-restricted cluster of differentiation (CD4)(CD) 4-positive helper $\mathrm{T}$ cells, which then activate $B$ cells and the humoral response. This is the mechanism by which most human vaccines induce immunity. This system cannot "see" inside cells, so antigens such as viral nucleoproteins that are produced intracellularly are not detected. The class I MHC system or the endogenous pathway is designed to identify intracellular antigens. All nucleated cells in the body express class I MHC molecules, which are located on the endoplasmic reticulum (ER). Intracellular antigens cleaved to 8- to 12 -amino-acid peptides are then transported into the ER, where they bind to MHC class I molecules. The MHC-antigen complex then travels 
through the Golgi apparatus and is expressed on the cell surface. This complex is recognized by class I MHC CD8-positive restricted cytotoxic $\mathrm{T}$ lymphocytes, which in turn are activated and attack infected cells (Fig. 1).

Influenza $A$ is infamous for its ever-changing envelope proteins. If one has been vaccinated against a particular strain, when the virus first enters the body neutralizing antibodies against the envelope will prevent intracellular infection and reproduction of the virus. Next year, when the virus returns, the envelope proteins will have changed and the antibodies from last year's vaccine no longer recognize the envelope's antigenic epitopes. Influenza A nucleoprotein does not change significantly from year to year, which may indicate that the virus cannot survive major changes in nucleoprotein stucture. If one were to try to utilize the nucleoprotein as a vaccine by means of traditional methods, the induced class II MHC humoral antibody response might not be effective against infection because antibody would not have access to the nucleoprotein in intact virions or cells. But if DNA coded for the nucleoprotein were used, the protein would be made intracellularly and processed through the endogenous pathway, and CD8-positive cytotoxic $T$ lymphocytes (CTLs) could attack and clear cells containing the virion (Fig. 1). Ulmer and colleagues placed the DNA sequence for influenza nucleoprotein in a plasmid, a circular piece of bacterial DNA. In front of the nucleoprotein sequence they placed a Rous sarcoma virus promoter (i.e., a DNA sequence that would tell the host cell to transcribe the nucleoprotein DNA sequence into messenger RNA). From there the host cells would, with luck, translate the messenger RNA into protein. When Ulmer et al. actually gave the plasmid vector to mice by injecting it into the quadriceps muscle, they were able to show that mice developed antibodies and cytotoxic $\mathrm{T}$ lymphocytes to the nucleoprotein. Next they demonstrated that mice immunized with the vector could survive what would otherwise be a lethal dose of influenza $\mathrm{A}$. This protection existed across different strains of influenza with different envelope proteins, something that would not occur with the usual influenza vaccines. The only difficulty with this work is that these investigators did not definitively demonstrate that CD8positive restricted CTLs were the mechanism for the observed protection against influenza infection. As indirect evidence for the primary role of CTLs, Ulmer et al. immunized mice with purified nucleoprotein; these mice exhibited high titers of nucleoprotein IgG antibody but could not survive a lethal dose of virus. This indicates that humoral antibodies were insufficient to protect the animals; therefore cell-mediated CTLs were probably responsible for the observed protection against viral infection.

These experiments may have important implications for developing a vaccine against hepatitis $\mathrm{C}$ virus (HCV). Some aspects of HCV infection, compared with those of HBV, suggest that traditional vaccine approaches may not be effective in inducing immunity against $\mathrm{HCV}$ (5). About $95 \%$ of persons who become infected with HBV generate an adequate immune response and clear the

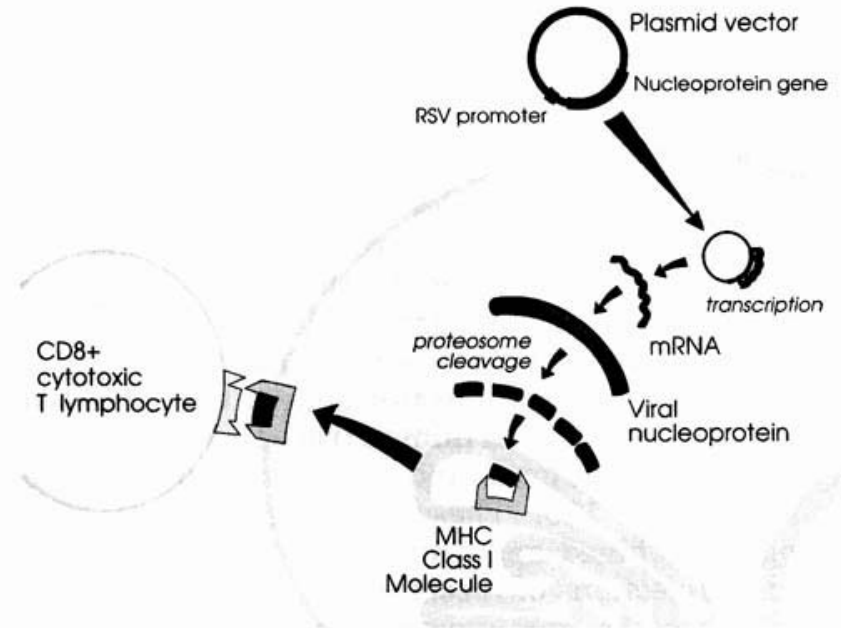

FIG. 1. The plasmid vector containing an RSV promoter and influenza A nucleoprotein gene is injected into muscle and taken up by cells. The nucleoprotein gene is transcribed into messenger RNA, which is translated into nucleoprotein. The protein is cleaved, enters the endoplasmic reticulum and binds to MHC class I molecules, which are then transported through the Golgi apparatus to the cell surface, where the complex activates CTLs.

virus. $\mathrm{HBs}$ antibody is considered neutralizing for HBV. Purified HBsAg is an effective vaccine against $\mathrm{HBV}-$ that is, the generation of HBs antibody is protective against infection. In HCV the story is quite different. More than $60 \%$ of persons infected with HCV become chronic carriers $(6,7)$. For whatever reason, most people cannot generate an adequate immune response to clear the virus, and most will be subject to some form of liver damage. Antibodies generated against the nucleocapsid core region, NS3 and NS4 of the HCV genome, are present in most people chronically infected with HCV; therefore these antibodies are not neutralizing $(8,9)$. HCV virus is detectable in most people who display these antibodies, and experiments in chimpanzees have shown that chimps can be reinfected with the same strain of HCV despite the presence of antibodies $(10,11)$. These data suggest that a humorally mediated vaccine may not protect against HCV infection. Chiron Corp. (Emeryville, CA), in conjunction with Ciba-Geigy (Basel, Switzerland) is developing a recombinant vaccine using $\mathrm{HCV}$ envelope glycoproteins and an undefined adjuvant. Their approach is a variation on traditional methodologies to evoke a humoral response to an antigen. Preliminary data were presented in May at the Triennial International Symposium on Viral Hepatitis and Liver Disease in Tokyo by Michael Houghton Ph.D. Of seven chimpanzees given the vaccine and then challenged with $\mathrm{HCV}$, five were judged to have remained infection-free on the basis of numerous parameters, including polymerase chain reaction. All control animals in the experiment became infected. Although these preliminary data are encouraging, proven efficacy in human subjects is a ways off, and theoretical concerns remain. The approach outlined by Ulmer et al. may prove to be a useful alternative for developing a vaccine to $\mathrm{HCV}$ if a traditional 
vaccine does not work or as adjuvant therapy in persons already infected with HCV to augment the immune response and promote clearance of the virus.

In closing, it should be noted that although current vaccines for HIV consist of recombinant gp 160 glycoprotein, Graham et al. are developing a human immunodeficiency virus vaccine using methods similar to those described here by inserting the gp 160 gene into vaccinia virus (HIVAC-1e; Bristol-Meyers Squibb, Seattle, WA) (12). HIVAC-1e is given subcutaneously; it promotes a local vaccinia infection similar to that raised by the smallpox vaccine. In human trials this vaccine has generated both humoral and cell-mediated immune responses to gp 160. These investigators are now studying a combined approach using HIVAC-1e virus followed by injections of recombinant gp 160 protein to produce a greater immunogenic response (13). This combined application is another approach that may lend itself to the prophylaxis or treatment of $\mathrm{HCV}$.

\section{W. MICHAEL MCDONNELL, M.D. Department of Internal Medicine University of Michigan Medical School Ann Arbor, Michigan 48109-0362}

\section{REFERENCES}

1. Mulligan RC. The basic science of gene therapy. Science 1993; 260:926-932

2. Cohen J. Naked DNA points way to vaccines. Science 1993;259: 1691-1692

3. Wolff JA, Malone RW, Williams P, et al. Direct gene transfer into mouse muscle in vitro. Science 1990;247:1465-1468.

4. Bolognesi DP. Fresh pathways to follow. Nature 1990;344: 818-819.

5. Lanzavecchia A. Identifying strategies for immune intervention. Science $1993 ; 260: 937-944$.

6. Omata M, Yokosuka O, Takano S, et al. Resolution of acute hepatitis $\mathrm{C}$ after therapy with natural beta interferon. Lancet 1991;338:914-915.

7. Alter MJ, Margolis HS, Krawczynski K, et al. The natural history of community acquired hepatitis $\mathrm{C}$ in the United States. N Engl J Med 1992;327:1899-1905.

8. Tanaka E, Kiyosawa K, Nakatsuji $Y$, et al. Clinical significance of antibodies to nonstructural and core proteins of hepatitis $C$ virus in posttransfusion hepatitis patients during long-term follow-up. J Med Virol 1993;39:318-324.

9. Okamoto H, Tsuda F, Machida A, et al. Antibodies against synthetic oligopeptides deduced from the putative core gene for the diagnosis of HCV infection. HePATOLOGY 1992;15:180-186.

10. Farci P, Alter HJ, Govindarajan S, et al. Lack of protective immunity against reinfection with hepatitis $\mathrm{C}$ virus. Science 1992;258:135-140.

11. Farci P, Alter HJ, Wong D, et al. A long-term study of HCV replication in non-A, non-B hepatitis. N Engl J Med 1991;325 98-104.

12. Graham BS, Belshe RB, Clements $M L$, et al. Vaccination of vaccinia-naive adults with HIV type I gp 160 recombinant vaccinia virus in a blinded, controlled, randomized clinical trial. J Infect Dis 1992;166:244-252.

13. Graham BS, Matthews TJ, Belshe RB, et al. Augmentation of HIV type 1 neutralizing antibody by priming with gp160 recombinant vaccinia and boosting with $\mathrm{rgp} 160$ in vaccinia-naive adults. $J$ Infect Dis 1993;167:533-537.

\section{CRYOGLOBULINEMIA AND HEPATATROPHIC VIRUSES}

Marcellin P., Descamps V., Martinot-Peignoux M., Larzul L., Xu L., Boyer N., Pham B.-N., Crickx B.,
Guillevin L., Belaich S., Erlinger S., and Benhamou $J . P$. Cryoglobulinemia with vasculitis associated with hepatitis C virus infection. Gastroenterology 1993;104: $272-277$.

\section{ABSTRACT}

Essential mixed cryoglobulinemia is frequently associated with chronic hepatitis. This report presents four cases of cryoglobulinemia with vasculitis associated with chronic hepatitis related to hepatitis $\mathrm{C}$ virus infection. Hepatitis $C$ virus infection was ascertained in the four patients by both the presence in the serum of anti-HCV antibodies detected by the fourantigen recombinant immunoblot assay and of $\mathrm{HCV}$ RNA detected by polymerase chain reaction. In two patients tested, anti-HCV antibodies were not detected after centrifugation in the purified cryoglobulin but were detected in the supernatant. HCV RNA was detected in the purified cryoglobulin in all four patients and was detected in the supernatant in three patients. In one patient receiving recombinant interferon alfa, serum aminotransferases normalized and cryoglobulin disappeared; in another patient receiving recombinant interferon alfa, serum aminotransferases remained high and the cryoglobulin persisted. The presence of HCV RNA in the cryoglobulin and the parallelism of the changes of the cryoglobulinemia and of the serum aminotransferases during recombinant interferon alfa administration suggest that HCV infection is responsible for the production of cryoglobulinemia and vasculitis. It is proposed that HCV infection is a cause of cryoglobulinemia associated with chronic hepatitis.

Agnello V., Chung R.T., Kaplan L.M. A role for hepatitis $\mathrm{C}$ virus infection in type II cryoglobulinemia. N Engl J Med 1992;327:1490-1495

\section{ABSTRACT}

Background. Type II cryoglobulinemia is a vasculitis characterized by cryoglobulins consisting of com. plexes of polyclonal IgG and monoclonal IgM rheumatoid factors. The cause of these immune complexes is unknown, though both the hepatitis B (HBV) and C (HCV) viruses have been suspected.

Methods. We studied 19 patients with Type II cryoglobulinemia for markers of $\mathrm{HCV}$ and $\mathrm{HBV}$ infection. Quantitative HCV antibody and RNA studies were performed on whole serum, cryoprecipitates, and supernatants.

Results. Eight patients (42 percent) had HCV antibodies, and 16 (84 percent) had HCV RNA. Of the 19 patients, 5 (26 percent) had HBV markers, but only 1 had evidence of active HBV infection. Control serum samples from nine patients with Type I cryoglobulinemia were negative for $\mathrm{HCV}$ antibody and HCV RNA. There was a close, although not exclusive, association of one type of rheumatoid factor (WA) with HCV RNA. HCV antibody and HCV RNA were concentrated approximately 10-fold and 1000-fold, respectively, in the Type II cryoglobulins examined.

Conclusions. Type II cryoglobulinemia is strongly associated with concomitant $\mathrm{HCV}$ infection and a high rate of false negative serologic tests. HCV virions and HCV antigen-antibody complexes are concentrated in the cryoprecipitates, most commonly in association with the WA type of rheumatoid factor, suggesting a 